Artículo

\title{
Productividad de quince cultivares tradicionales de papa Phureja en ocho ambientes distintos
}

\author{
Alejandro Seminario Cunya ${ }^{1}$ \\ Pablo Huerta Fernández ${ }^{2 \S}$ \\ Víctor Vásquez Arce A $^{3}$ \\ Juan Seminario Cunya ${ }^{4}$ \\ Marco Honorio Acosta ${ }^{5}$ \\ Antonio Huerta Fernández ${ }^{6}$ \\ ${ }^{1}$ Universidad Nacional Autónoma de Chota. Jr. Los Andes 184, Barrio San Martín Cajamarca, Perú. CP. \\ 054. (aseminarioc@unach.edu.pe). ${ }^{2}$ Universidad Nacional Agraria La Molina. Calle Madrid 116, \\ Departamento 101, Lima, Perú. CP. 18. Tel. 51 971129290. (apablohuerta@gmail.com). ${ }^{3}$ Universidad \\ Nacional de Cajamarca. Jr. Los Fresnos 139, Urb. El Ingenio, Cajamarca, Perú. (vvarce3@ gmail.com). \\ ${ }^{4}$ Universidad Nacional de Cajamarca. Jr. Rafael Olascoaga 141, Urb. Horacio Cevallos, Cajamarca, Perú. \\ (jfseminario@yahoo.es). ${ }^{5}$ Universidad Privada del Norte. Av. Del Ejército núm. 920, Trujillo, La Libertad, \\ Perú. CP. 1309. Tel. 51 948673737. (mahonac@hotmail.com). ${ }^{6}$ Universidad de Lleida. Plaza de Victor \\ Siurana 1. Lleida, España. CP. 25003. (antoniohuerta2010@gmail.com). \\ ${ }^{\S}$ Autor para correspondencia: apablohuerta@gmail.com.
}

\section{Resumen}

Se evaluó la productividad de tubérculos frescos y de materia seca de 15 cultivares tradicionales de papa del grupo Phureja de Cajamarca, en ocho ambientes distintos. Las siembras se realizaron en el valle de Cajamarca. Se usó el diseño de bloques completos al azar, con 15 tratamientos y tres repeticiones. La siembra se realizó a $0.9 \mathrm{~m}$ entre surcos y $0.4 \mathrm{~m}$ entre plantas. Se aplicó $5 \mathrm{t} \mathrm{ha} \mathrm{C}^{-1} \mathrm{de}$ humus de lombriz y un fertilizante compuesto (15-24 -14 de N, P y K), a razón de $300 \mathrm{~kg} \mathrm{ha}^{-1}$. A la cosecha se tomaron ocho plantas por tratamiento y se evaluaron las variables relacionadas con el rendimiento. Se encontraron diferencias estadísticas significativas entre cultivares para las variables: peso total de tubérculos, peso de tubérculos comerciales, número total de tubérculos, número de tubérculos comerciales, altura de planta, número de tallos y materia seca. Los cultivares 'Chachapoyana', 'Montañera', 'Blanca' y 'Shoga', fueron los mejores en rendimiento de tubérculos frescos y los cultivares 'Piña amarilla', 'Shoga' y 'Porpora', los mejores en materia seca. Los ambientes presentaron diferencias estadísticas significativas paras las variables NTT, NTC, AP y NT.

Palabras clave: andina, ecología, producción.

Recibido: abril de 2021

Aceptado: junio de 2021 


\section{Introducción}

La papa (Solanum tuberosum L.) del grupo Phureja es importante en el Perú y otros países andinos por sus atributos para el mejoramiento genético, el mercado y el consumo en fresco y procesado (Piñeros, 2009; Rozo y Ramírez, 2011). Por sus ventajas comparativas esta papa es objeto de estudios desde diversos enfoques.

En los últimos años, los estudios se han dirigido especialmente a conocer sus ventajas desde el punto de vista físico-químico (contenido de materia seca, proteína, almidón, azúcares totales y azúcares reductores) (Cerón-Lasso et al., 2018). Otros estudios valoran el contenido de metabolitos secundarios (carotenoides, fenoles y antocianinas), la vitamina $\mathrm{C}$ y el poder antioxidante de este conjunto de compuestos (Bonierbale et al., 2009; Díaz et al., 2014; Molina et al., 2015; ParraGalindo et al., 2016). También se han estudiado los macro y micronutrientes, especialmente Ca, Fe y Zn (Peña et al., 2015). Del mismo modo, se estudiaron sus ventajas para la industria alimentaria (Rivera et al., 2011; Cruz et al., 2016; Alarcón et al., 2016) y de modo particular, los almidones (Martínez et al., 2015).

Colombia es el país que mejor ha aprovechado las ventajas de esta papa, tanto para el mercado interno como externo. Hace algunos años, sus exportaciones en diversos derivados ascendían a más de $1000 \mathrm{t} \mathrm{año}^{-1}$. En el Perú no se han aprovechado sus bondades, por el contrario, esta papa estaría perdiéndose de forma acelerada (Zimmerer, 1991; Haan, 2009; Seminario y Zarpán, 2011). Sin embargo, las exploraciones de los últimos años indican que, en el norte peruano, particularmente en Cajamarca, existen diversos cultivares que se diferencian principalmente por la forma, color y tamaño de los tubérculos y la forma y color de las flores. Estos son sembrados en extensiones pequeñas por agricultores tradicionales, con fines de autoconsumo y de mercado (Seminario y Zarpán, 2011; Seminario et al., 2019).

Los estudios sobre rendimiento de tubérculos frescos de la papa Phureja varían ampliamente. Así, por ejemplo, con cultivares tradicionales -no mejorados-, se reportan rendimientos de 11.8 a $23 \mathrm{t}$ ha $^{-1}$ (Bautista et al., 2012), 13.9 a 21.9 t ha $^{-1}$ (Gómez-García, 2017) y 6.2 a 27.4 t ha $^{-1}$ (Seminario et al., 2017). Por otro lado, con cultivares mejorados mediante cruzamiento y selección se mencionan rendimientos mayores. Así se tiene, 35 a $41.8 \mathrm{t} \mathrm{ha}^{-1}$ (León y Monteros, 2010) $31.7 \mathrm{a}$ $34.3 \mathrm{t} \mathrm{ha}^{-1}$ (Santa María et al., 2010) y 32.9 a 37.4 t ha-1 (Rodríguez et al., 2014).

Por este motivo, es importante estudiar el potencial productivo de los cultivares de cada región, para seleccionar aquellos que destacan por esta característica y realizar estudios sobre el aprovechamiento en fresco y procesado y su intervención en la generación de variedades mejoradas. En este sentido, el objetivo de la presente investigación fue evaluar los componentes del rendimiento y la productividad de 15 cultivares tradicionales del grupo Phureja de Cajamarca, en ocho ambientes distintos, misma localidad, pero en diferentes años y meses de siembra.

\section{Materiales y métodos}

Se usaron 15 cultivares nativos del grupo Phureja (Cuadro 1) de la colección de trabajo del Programa de Raíces y Tubérculos Andinos de la Universidad Nacional de Cajamarca (PRTAUNC). Estos cultivares proceden de la región Cajamarca, fueron caracterizados morfológicamente y se encuentran catalogados formalmente (Seminario et al., 2019). 
Cuadro 1. Cultivares de papa del grupo Phureja de Cajamarca, usados en la investigación.

\begin{tabular}{cccc}
\hline Cultivar & Abreviatura & Procedencia & Forma tubérculo \\
\hline 'Chachapoyana' & Cha & Bambamarca & Oblongo \\
'Montañera' & Mon & Bambamarca & Redondo \\
'Blanca' & Bla & Bambamarca & Comprimido \\
'Shoga' & Sho & Jesús & Oblongo \\
'Piña amarilla' & Pam & Jesús & Comprimido \\
'Mulla' & Mul & Jesús & Elíptico \\
'Poropora' & Por & Bambamarca & Oblongo \\
'Limeña' & Lim & Chota & Comprimido \\
'Amarila mahuay' & Amh & Jesús & Comprimido \\
'Limeña hachuma' & Lih & Jesús & Comprimido \\
'Huagalina' & Hua & Hualgayoc & Elíptico \\
'Amarilla' & Ama & Jesús & Comprimido \\
'Yuquilla' & Yuq & Jesús & Elíptico \\
'Huevo de ruco' & Hur & Bambamarca & Obovado \\
'Huamantanga' & Hut & La Encañada & Ovado \\
\hline
\end{tabular}

El suelo donde se realizaron las siembras corresponde a un lote de aproximadamente 0.7 ha, ubicado en el Valle de Cajamarca, a $2650 \mathrm{msnm}$. En este lote, las siembras se realizaron en un sistema de rotación de cultivos que incluye yacón, papa, descanso, papa, yacón, descanso. El suelo es de textura franco arcillosa y los promedios de ocho muestras tomadas en años y puntos diferentes, indicaron que el $\mathrm{pH}$ es neutro a ligeramente ácido, el contenido de materia orgánica (MO) es bajo y los de fósforo $(\mathrm{P})$ disponible y potasio $(\mathrm{K})$ disponible fueron de medios a altos (Cuadro 2).

Cuadro 2. Valores de $\mathrm{pH}$, MO, $\mathrm{P}$ disponible y $\mathrm{K}$ disponible del suelo donde se realizaron las siembras.

\begin{tabular}{cccccc}
\hline Número & pH & MO & P disponible & K disponible & Laboratorio/código \\
\hline 1 & 7.3 & 3.8 & 13.8 & 213.3 & Tec. Des. Agr. JD. SRL.JD15-0122 \\
2 & 6.9 & 4 & 11.8 & 239.5 & Tec. Des. Agr. JD. SRL. JD1700 \\
3 & 6.6 & 3.2 & 23.8 & 295 & Est. Exp. BI, INIA. SU0287-EEBI \\
4 & 6.4 & 1.5 & 30.5 & 300 & Est. Exp. BI, INIA. SU1253-EEBI \\
5 & 6.2 & 2.1 & 21.5 & 290 & Est. Exp. BI, INIA. SU0787-EEBI \\
6 & 7.5 & 3 & 15.2 & 237 & UNALM, 9431. \\
7 & 5.7 & 2.4 & 19 & 265 & Est. Exp. BI, INIA. SU1252 - EEBI. \\
8 & 6.6 & 2.4 & 35.5 & 310 & Est. Exp. BI, INIA. SU0794-EEBI. \\
Promedio & 6.6 & 2.8 & 21.4 & 268.7 & \\
\hline
\end{tabular}

Tec. Des. Agr= Tecnología y Desarrollo Agrícola; Est. Exp. BI, INIA= Estación Experimental Baños del Inca, Instituto Nacional de Innovación Agraria; UNALM= Universidad Nacional Agraria La Molina. 
Durante ocho años se sembraron los 15 cultivares en fechas diferentes Cuadro 3. Las variables climáticas básicas y la temporada (lluvia/estiaje) bajo las cuales se realizaron las siembras se presentan en la Cuadro 4. La diferencia más notable entre temporadas es la cantidad de lluvia, sin embargo, esta falta de lluvia en las siembras de época de estiaje se superó con la aplicación de riegos por gravedad. La diferencia en temperatura promedio fue de $0.55^{\circ} \mathrm{C}$, en temperatura mínima de $2.6^{\circ} \mathrm{C}$ y en horas de sol de $1.7 \mathrm{~h}$.

Cuadro 3. Fechas de siembra y cosechas y temporada de los ensayos.

\begin{tabular}{cccc}
\hline Número & Fecha siembra & Fecha cosecha & Temporada \\
\hline 1 & $20-12-10$ & $18-04-11$ & Lluvia \\
2 & $30-04-11$ & $03-09-11$ & Estiaje \\
3 & $28-11-11$ & $10-04-12$ & Lluvia \\
4 & $25-05-12$ & $07-10-12$ & Estiaje \\
5 & $01-09-12$ & $09-01-13$ & Estiaje/lluvia \\
6 & $25-06-14$ & $12-11-14$ & Estiaje \\
7 & $25-11-15$ & $31-03-16$ & Lluvia \\
8 & $27-07-17$ & $05-12-17$ & Estiaje/lluvia \\
\hline
\end{tabular}

En la siembra de cada año, cada cultivar ocupaba un surco con 10 plantas. De este modo cada siembra (en ambiente diferente), constituye un bloque o repetición. Se usó el diseño experimental de bloques completos al azar, con 15 tratamientos (cultivares). Los distanciamientos de siembra fueron $0.9 \mathrm{~m}$ entre surcos por $0.4 \mathrm{~m}$ entre plantas (27 778 plantas ha ${ }^{-1}$ ). En cada siembra se aplicó $5 \mathrm{t} \mathrm{ha}^{-1}$ de humus de lombriz y $300 \mathrm{~kg} \mathrm{ha}^{-1}$ del fertilizante compuesto Compomaster papa sierra, que contiene $15 \%, 24 \%$ y $14 \%$ de nitrógeno, fósforo y potasio, respectivamente.

A la cosecha se evaluaron ocho plantas, desechando las plantas de los extremos para evitar el efecto de borde. El manejo cultural de los cultivos consistió en deshierbes, riegos y control sanitario, según las necesidades específicas del cultivo y de acuerdo con la temporada, siguiendo las recomendaciones para este tipo de papa, establecidas por el PRTA-UNC y Navas y Díaz (2012).

Cuadro 4. Valores de las variables climáticas básicas durante los meses y años en los cuales se realizaron las siembras.

\begin{tabular}{|c|c|c|c|c|c|c|c|c|c|c|c|c|c|c|c|c|}
\hline \multirow{2}{*}{ Variable } & \multicolumn{4}{|c|}{ Siembra 1: 2010} & \multicolumn{4}{|c|}{ Siembra 2: 2011} & \multicolumn{3}{|c|}{ Siembra 3: 2011} & \multicolumn{5}{|c|}{ Siembra 4: 2012} \\
\hline & $\begin{array}{l}\text { Ene- } \\
2011\end{array}$ & $\begin{array}{l}\text { Feb- } \\
2011\end{array}$ & $\begin{array}{l}\text { Mar- } \\
2011\end{array}$ & $\begin{array}{l}\text { Abr- } \\
2011\end{array}$ & $\begin{array}{l}\text { May- } \\
2011\end{array}$ & $\begin{array}{l}\text { Jun- } \\
2011\end{array}$ & $\begin{array}{l}\text { Jul- } \\
2011\end{array}$ & $\begin{array}{l}\text { Ago- } \\
2011\end{array}$ & $\begin{array}{l}\text { Dic- } \\
2011\end{array}$ & $\begin{array}{l}\text { Ene- } \\
2012\end{array}$ & $\begin{array}{l}\text { Feb- } \\
2012\end{array}$ & $\begin{array}{l}\text { Mar- } \\
2012\end{array}$ & $\begin{array}{l}\text { Jun- } \\
2012\end{array}$ & $\begin{array}{l}\text { Jul- } \\
2012\end{array}$ & $\begin{array}{l}\text { Ago- } \\
2012\end{array}$ & $\begin{array}{l}\text { Sep- } \\
2012\end{array}$ \\
\hline $\mathrm{Pp}(\mathrm{mm})$ & 76.6 & 73.3 & 125.2 & 102 & 16.7 & 0.4 & 8.3 & 0 & 110.6 & 154.2 & 155.3 & 127.8 & 0.9 & 0 & 2.6 & 19 \\
\hline $\mathrm{T}\left({ }^{\circ} \mathrm{C}\right)$ & 14.6 & 14.2 & 3.9 & 14.4 & 14.4 & 14.2 & 13.9 & 4.4 & 14.9 & 14.2 & 14.4 & 15 & 13.6 & 13.8 & 14.2 & 14.6 \\
\hline $\begin{array}{c}\mathrm{T} \max \\
\left({ }^{\circ} \mathrm{C}\right)\end{array}$ & 21 & 20.8 & 20.2 & 20.9 & 21.7 & 21.7 & 21.2 & 22.2 & 21 & 20.9 & 20.6 & 21.3 & 21.6 & 22 & 22.1 & 21.9 \\
\hline $\begin{array}{l}\mathrm{T} \min \\
\left({ }^{\circ} \mathrm{C}\right)\end{array}$ & 9.1 & 8.5 & 8.5 & 9.4 & 6.6 & 6.1 & 5.9 & 6 & 9.8 & 7.6 & 9.3 & 9.9 & 5 & 4.8 & 5.5 & 6.6 \\
\hline $\mathrm{HR}(\%)$ & 71 & 77 & 72 & 70 & 62 & 61 & 62 & 60 & 67 & 64 & 68 & 71 & 63 & 58 & 57 & 62 \\
\hline $\begin{array}{c}\text { Horas } \\
\text { sol }\end{array}$ & 4.5 & 4.1 & 3.5 & 5.1 & 5.8 & 6.6 & 6.4 & 8.3 & 4.7 & 5 & 5.5 & 4.1 & 8 & 7.8 & 8.7 & 5.8 \\
\hline
\end{tabular}




\begin{tabular}{|c|c|c|c|c|c|c|c|c|c|c|c|c|c|c|c|c|}
\hline \multirow[b]{2}{*}{ Variable } & \multicolumn{4}{|c|}{ Siembra 5: 2012} & \multicolumn{4}{|c|}{ Siembra 6: 2014} & \multicolumn{4}{|c|}{ Siembra 7: 2015} & \multicolumn{4}{|c|}{ Siembra 8: 2017} \\
\hline & $\begin{array}{l}\text { Sep- } \\
2012\end{array}$ & $\begin{array}{l}\text { Oct- } \\
2012\end{array}$ & $\begin{array}{l}\text { Nov- } \\
2012\end{array}$ & $\begin{array}{l}\text { Dic- } \\
2012\end{array}$ & $\begin{array}{l}\text { Jun- } \\
2014\end{array}$ & 20 & & $\begin{array}{l}\text { Oct- } \\
2014\end{array}$ & $\begin{array}{l}\text { Dic- } \\
2015\end{array}$ & $\begin{array}{l}\text { Ene- } \\
2016\end{array}$ & $\begin{array}{l}\text { Feb- } \\
2016\end{array}$ & $\begin{array}{l}\text { Mar- } \\
2016\end{array}$ & $\begin{array}{l}\text { Ago- } \\
2017\end{array}$ & $\begin{array}{l}\text { Sep } \\
201\end{array}$ & $\begin{array}{l}\text { Oct- } \\
2017\end{array}$ & $\begin{array}{l}\text { Nov- } \\
2017\end{array}$ \\
\hline$(\mathrm{mm})$ & 19 & 83 & 120 & 58.9 & 1.9 & 3.8 & 28.5 & 26.6 & 39.5 & 27 & 85.3 & 12.3 & 20.9 & 21.5 & 65.3 & 63.2 \\
\hline $\mathrm{T}^{\circ} \mathrm{C}$ & 14.6 & 10 & 15.4 & 15.3 & 14.4 & 14.3 & .7 & 15.4 & 16.5 & 16.5 & 16.4 & 16.2 & 14.5 & 15.3 & 15.7 & 15.7 \\
\hline $\begin{array}{c}\mathrm{T} \max \\
\left({ }^{\circ} \mathrm{C}\right)\end{array}$ & 21.9 & 21.2 & 21.6 & 22.1 & 22.3 & 21.5 & 21.9 & 22.4 & 22.9 & 23.7 & 22.3 & 22.5 & 22 & 21.1 & 22.5 & 23.2 \\
\hline $\begin{array}{l}\mathrm{T} \min \\
\left({ }^{\circ} \mathrm{C}\right)\end{array}$ & 6.6 & 9.5 & 10.2 & 9 & 5.8 & 5.9 & 7.6 & 8.7 & 10.4 & 10.5 & 11.8 & 10.6 & 6.5 & 8.3 & 8.8 & 8.4 \\
\hline $\mathrm{HR}(\%)$ & 62 & 67 & 66 & 62 & 57 & 55 & 60 & 57 & 67 & 69 & 70 & 69 & 60 & 54 & 54 & 58 \\
\hline $\begin{array}{c}\text { Horas } \\
\text { sol }\end{array}$ & 5.8 & 4.7 & 5.6 & 6.3 & 7.9 & 6.7 & 5.6 & 5.1 & 4.7 & 6.8 & 4 & 4 & 7.3 & 5.2 & 6.5 & 6.5 \\
\hline
\end{tabular}

Cada cuatro columnas corresponden a los cuatro meses (abreviados por la primera letra) de cada siembra. $\mathrm{T}{ }^{\circ} \mathrm{C}=$ corresponde a temperatura promedio diaria. El número de cada siembra corresponde al número de siembra del Cuadro 3. En gris las siembras en temporada de estiaje. Estación Meteorológica Augusto Weberbauer (2 $650 \mathrm{msnm})$, convenio UNC-SENAMHI.

Las variables evaluadas fueron peso total de tubérculos (PTT); peso de tubérculos comerciales (PTC); número total de tubérculos (NTT); número de tubérculos comerciales (NTC); altura de planta (AP); número de tallos y el rendimiento de materia seca de los tubérculos. Esta materia seca se determinó tomando como base el porcentaje de materia seca de los cultivares obtenido durante la caracterización Seminario et al. (2019). La discriminación de los tubérculos comerciales se realizó con la clasificación de Pérez et al. (2008) y los criterios establecidos por Seminario et al. (2018) en tubérculos redondos y comprimidos: diámetro $\geq 2 \mathrm{~cm}$ y en tubérculos oblongos, elípticos y obovados y ovados: $\mathrm{L}+\mathrm{A} / 2 \geq 2 \mathrm{~cm}$.

Los datos de las variables en estudio fueron analizados mediante el programa SAS versión 9.4. Primero se realizó el análisis de varianza (Anova) y luego, según la significación de esta prueba, se realizó la prueba de rango múltiple de Duncan. Además, se hicieron correlaciones entre las variables relacionadas con el rendimiento y el peso total de tubérculos. El rendimiento de tubérculos frescos por hectárea se calculó mediante la ecuación de Hay y Walker (1989): peso fresco de tubérculos (ha) = densidad de plantación x número de tubérculos por planta x peso promedio del tubérculo. El rendimiento de materia seca por hectárea se determinó multiplicando el peso promedio de la materia seca de la planta por el número de plantas por hectárea.

\section{Resultados y discusión}

\section{Rendimiento de tubérculos frescos y variables relacionadas}

Se encontraron diferencias estadísticas altamente significativas $(p>0.01)$ para los cultivares en las seis variables estudiadas (Cuadro 5); es decir, existen diferencias reales entre los promedios de dichas variables. Estas diferencias se explican, probablemente, por la variabilidad genética de los cultivares en estudio. 
Cuadro 5. Cuadrados medios y significación del Anova, para el peso total de tubérculos por planta (PTT) y variables relacionadas de 15 cultivares de papa del grupo Phureja de Cajamarca, en ocho ambientes.

\begin{tabular}{ccccccccc}
\hline $\begin{array}{c}\text { Fuente de } \\
\text { variación }\end{array}$ & $\begin{array}{c}\text { Grados de } \\
\text { libertad }\end{array}$ & PTT $(\mathrm{g})$ & PTC $(\mathrm{g})$ & NTT & NTC & AP & NT & MS \\
\hline Bloque & 7 & $7301.12 \mathrm{~ns}$ & $2363.82 \mathrm{~ns}$ & $4.27^{* *}$ & $0.89^{* *}$ & $0.89^{* *}$ & $0.21^{* *}$ & $408.0948^{*}$ \\
Cultivares & 14 & $46043.15^{\text {** }}$ & $27633.35^{* *}$ & $3.23^{* *}$ & $0.37^{* *}$ & $0.37^{* *}$ & $0.24^{* *}$ & $2190.6406^{* *}$ \\
Error & 98 & 3581.32 & 2520.83 & 1.13 & 0.18 & 0.18 & 0.07 & 185.1059 \\
Total & 119 & & & & & & & \\
CV $(\%)$ & & 21.7 & 27.6 & 26.9 & 20 & 20 & 13.5 & 21.9 \\
\hline
\end{tabular}

PTT= peso total de tubérculos $(\mathrm{g})$; PTC= peso de tubérculos comerciales $(\mathrm{g})$; NTT= número total de tubérculos, NTC= número de tubérculos comerciales; $\mathrm{AP}=$ altura de planta $(\mathrm{cm}) ; \mathrm{NT}=$ número de tallos; $\mathrm{CV}=$ coeficiente de variación $(\%) .{ }^{*}=$ significativo ${ }^{* *}=$ altamente significativo; $\mathrm{ns}=$ no significativo; $\mathrm{MS}=$ materia seca.

También se encontró significación estadística ( $p>0.01)$ para los ambientes (repeticiones) en cuatro variables relacionadas con el rendimiento (NTT, NTC, AP y NT), lo cual sugiere que los ambientes, en donde se realizaron los ensayos (años y meses diferentes) ejercieron influencia en los componentes evaluados, de modo diferenciado. Los coeficientes de variación variaron entre $13.5 \%$ y $27.6 \%$, éstos valores se consideran aceptables para las condiciones de campo en donde se realizaron los ensayos (Vásquez, 2014). Era de esperarse esta influencia debido a que las siembras se realizaron en diferentes temporadas (lluvia y estiaje) y en donde las temperaturas, las precipitaciones y las horas de sol, variaron de modo significativo (Cuadro 4).

En pruebas anteriores con cultivares del mismo grupo, se encontraron diferencias estadísticas para la mayoría de las variables aquí evaluadas (Pérez et al., 2008; Rozo y Ñustez, 2011; Rojas y Seminario, 2014; Seminario et al., 2017; Seminario et al., 2018). Estas similitudes son importantes porque permiten identificar, las variables cuantitativas que diferencian a los cultivares y también identificar los componentes básicos del rendimiento como son el número tubérculos y el peso promedio de tubérculos por planta los que combinados con la densidad de plantación permiten estimar el rendimiento por hectárea (Hay y Walker, 1989).

Según la prueba de Duncan $(\alpha=0.05)$ (Cuadro 6) se observa que los cultivares 'Chachapoyana', 'Montañera', 'Blanca' y 'Shoga', son similares estadísticamente y destacan por el peso total de tubérculos (PTT) y peso de tubérculos comerciales (PTC) respecto a los once cultivares restantes.

Cuadro 6. Prueba de rango múltiple de Duncan $(\alpha=0.05)$ para el peso total de tubérculos y variables relacionadas de 15 cultivares de papa del grupo Phureja de Cajamarca, en ocho ambientes.

\begin{tabular}{cccccccc}
\hline Cultivar & PTT $(\mathrm{g})$ & PTC $(\mathrm{g})$ & NTT & NTC & AP $(\mathrm{cm})$ & NT & MS $(\mathrm{g})$ \\
\hline Cha & $406.7 \mathrm{a}$ & $277.3 \mathrm{a}$ & $16.4 \mathrm{ab}$ & $6.1 \mathrm{ab}$ & $56.6 \mathrm{~d}$ & $5.1 \mathrm{a}$ & $81.3 \mathrm{ab}$ \\
Mon & $391.1 \mathrm{a}$ & $277.8 \mathrm{a}$ & $10.3 \mathrm{c}$ & $6.9 \mathrm{a}$ & $60.4 \mathrm{dc}$ & $5 \mathrm{~b}$ & $74.3 \mathrm{abcd}$ \\
Bla & $382.8 \mathrm{a}$ & $267.9 \mathrm{a}$ & $16.4 \mathrm{abc}$ & $4.9 \mathrm{abcd}$ & $62.1 \mathrm{dc}$ & $4.7 \mathrm{abcd}$ & $91.8 \mathrm{a}$ \\
Sho & $367.2 \mathrm{a}$ & $240.9 \mathrm{a}$ & $9.6 \mathrm{c}$ & $3.2 \mathrm{~d}$ & $67.5 \mathrm{bc}$ & $4.2 \mathrm{bcde}$ & $88.1 \mathrm{ab}$ \\
Pam & $302.7 \mathrm{~b}$ & $185.3 \mathrm{~b}$ & $22.9 \mathrm{ab}$ & $5.6 \mathrm{abc}$ & $60.4 \mathrm{dc}$ & $3.6 \mathrm{cde}$ & $66.6 \mathrm{bcd}$ \\
\hline
\end{tabular}




\begin{tabular}{cccccccc}
\hline Cultivar & PTT $(\mathrm{g})$ & PTC $(\mathrm{g})$ & NTT & NTC & AP $(\mathrm{cm})$ & NT & MS $(\mathrm{g})$ \\
\hline Mul & $269.3 \mathrm{bc}$ & $174.2 \mathrm{bc}$ & $17 \mathrm{abc}$ & $4.3 \mathrm{bcd}$ & $70.6 \mathrm{ab}$ & $3.1 \mathrm{e}$ & $61.6 \mathrm{cdef}$ \\
Por & $267.5 \mathrm{bcd}$ & $178.8 \mathrm{bc}$ & $22.8 \mathrm{ab}$ & $4.3 \mathrm{abcd}$ & $57 \mathrm{~d}$ & $3.7 \mathrm{de}$ & $64.2 \mathrm{cde}$ \\
Lim & $255 \mathrm{bcde}$ & $125.5 \mathrm{cde}$ & $11.7 \mathrm{bc}$ & $5.5 \mathrm{abc}$ & $59.7 \mathrm{dc}$ & $4.5 \mathrm{abcde}$ & $58.6 \mathrm{cdefg}$ \\
Amh & $228.5 \mathrm{cde}$ & $177.5 \mathrm{bc}$ & $11.5 \mathrm{bc}$ & $4.9 \mathrm{abcd}$ & $62.8 \mathrm{dc}$ & $3.8 \mathrm{bcde}$ & $61.7 \mathrm{cdef}$ \\
Lih & $227 \mathrm{cde}$ & $154.1 \mathrm{bcde}$ & $10.3 \mathrm{bc}$ & $4.8 \mathrm{abcd}$ & $65 \mathrm{bc}$ & $3.4 \mathrm{de}$ & $52.2 \mathrm{defg}$ \\
Hua & $223.2 \mathrm{cde}$ & $129.7 \mathrm{bcde}$ & $18.7 \mathrm{abc}$ & $4.9 \mathrm{abcd}$ & $62.1 \mathrm{dc}$ & $4.8 \mathrm{abc}$ & $42.4 \mathrm{efg}$ \\
Ama & $212.6 \mathrm{cde}$ & $171.3 \mathrm{bcd}$ & $20.1 \mathrm{abc}$ & $5.8 \mathrm{ab}$ & $76.3 \mathrm{a}$ & $5.4 \mathrm{a}$ & $57.4 \mathrm{defg}$ \\
Yuq & $200 \mathrm{de}$ & $146.2 \mathrm{bcde}$ & $22.3 \mathrm{ab}$ & $4.9 \mathrm{abcd}$ & $60.6 \mathrm{dc}$ & $4.6 \mathrm{abcd}$ & $39.8 \mathrm{fg}$ \\
Hur & $199 \mathrm{de}$ & $116.3 \mathrm{de}$ & $26.5 \mathrm{a}$ & $3.4 \mathrm{~cd}$ & $48 \mathrm{e}$ & $4.5 \mathrm{abcde}$ & $51.7 \mathrm{defg}$ \\
Hut & $197.8 \mathrm{e}$ & $100.8 \mathrm{e}$ & $20.2 \mathrm{abc}$ & $4.7 \mathrm{abcd}$ & $66.2 \mathrm{bc}$ & $4.7 \mathrm{abcd}$ & $37.6 \mathrm{~g}$ \\
\hline
\end{tabular}

El cultivar 'Chachapoyana' ocupa el primer lugar en cuanto a los componentes número total de tubérculos (NTT), número de tubérculos comerciales (NTC) y número de tallos (NT). Los cultivares 'Huagalina', 'Amarilla' y 'Yuquilla' no muestran diferencias estadísticas significativas para las variables NTT, NTC y NT. Con respecto a la altura de planta (AP) los cultivares Amarilla y Mulla no difieren estadísticamente, pero superan a los trece cultivares restantes.

La superioridad que muestran ciertos cultivares del grupo en estudio, con respecto a los componentes principales del rendimiento como el número y peso total de tubérculos $\mathrm{y}$, número y peso de tubérculos comerciales, constituye una ventaja que debe ser aprovechada para realizar pruebas de rendimiento específicas, con el propósito de identificar los cultivares más productivos de la región.

En estudios previos con cultivares del mismo grupo y de la región Cajamarca, han destacado otros cultivares en los componentes básicos del rendimiento (NTT, NTC, PTT y PTC). Así, se mencionan como los mejores: 'Roja-2', 'Piña amarilla' y 'Montañera-3' (Rojas y Seminario (2014). Roja-2, 'Amarilla mahuay', 'Piña amarilla', 'Amarilla', 'Montañera-3' y 'Porpora' (Seminario et al., 2017). 'Blanca amarilla', 'Limeña huachuma', 'Llanqueja', 'Amarilla redonda' y 'Piña amarilla' (Seminario et al., 2018). Estos cultivares élite se encuentran debidamente caracterizados morfológicamente (Seminario et al., 2019) y deben ser analizados mediante estudios específicos.

\section{Rendimiento de materia seca $\left(\mathrm{g} \mathrm{planta}^{-1}\right)$ de los cultivares}

Se encontró alta significación estadística para la fuente de variación cultivares, lo que significa que existen diferencias reales entre los promedios de la materia seca de los 15 cultivares. Del mismo modo, hubo significación estadística al 5\% de probabilidades para bloques (Cuadro 5), lo cual indica que hubo efecto ambiental en la variabilidad del contenido de materia seca en $\mathrm{g}$ planta ${ }^{-1}$. Es decir, el contenido de materia seca es un carácter controlado genéticamente y es afectado por las condiciones del medio y por la interacción genotipo x ambiente (Vásquez, 1988; Hay y Walker, 1989). El coeficiente de variación fue de $21.9 \%$, un valor aceptable para las condiciones de campo, donde se realizó el experimento. 
La prueba de rango múltiple Tukey $(\alpha=0.05)$ (Cuadro 6) indicó que no hay diferencias estadísticas entre los cultivares 'Montañera', 'Chachapoyana-1', 'Shoga' y 'Blanca', cuyos promedios varían entre 74.28 g y $91.88 \mathrm{~g}$, destacando el cultivar 'Blanca' con el más alto rendimiento de materia seca (91.888 $\left.\mathrm{g} \mathrm{planta}^{-1}\right)$. Se encontró un segundo grupo de cultivares formado por 'Piña amarilla', 'Poropora', 'Amarilla mahuay', 'Mulla' y 'Limeña', con promedios que varían entre $66.613 \mathrm{~g}$ planta $^{-1}$ y 58.638 g planta $^{-1}$ de materia seca, pero sin diferencia estadística entre ellos.

La variación en el rendimiento de materia seca entre los 15 cultivares (37.58 g planta ${ }^{-1}$ hasta 91.88 $\mathrm{g} \mathrm{planta}^{-1}$ ) se explica porque se trata de genotipos diferentes y también por el efecto ambiental. Se conoce que el contenido de materia seca es un carácter poligénico, altamente heredable y de baja interacción con el ambiente (Vásquez, 1988; Martínez y Ligarreto, 2005; Bautista et al., 2012). Diversos estudios han mostrado que en estos cultivares se espera que la mayor proporción de materia seca se asigne a los tubérculos, lo cual queda demostrado con el índice de cosecha (IC). Por lo general, los valores del IC son superiores a 40\% (Rojas y Seminario, 2014, Saldaña et al., 2015; Seminario et al., 2019).

Siendo así, es necesario conocer cuáles son los cultivares que tienen mayor producción de materia seca en los tubérculos, los cuales tienen ventaja para el consumo en fresco y para el procesamiento. El mayor contenido de materia seca implica consistencia harinosa, apreciada por el consumidor, menor absorción de aceite en las frituras y mayor rendimiento en fécula, puré, polvo, hojuelas y papa frita (Andrade, 1997; Ligarreto y Suarez, 2003).

\section{Rendimiento de tubérculos frescos $\left(\mathrm{t} \mathrm{ha}^{-1}\right)$ y de materia seca de los tubérculos $\left(\mathrm{t} \mathrm{ha} \mathrm{a}^{-1}\right)$}

Los rendimientos de tubérculos frescos por planta $\left(\mathrm{g} \mathrm{planta}^{-1}\right)$, significan 5 a $11 \mathrm{t} \mathrm{ha}^{-1}$ de tubérculos frescos (Figura 1). Estos rendimientos se pueden considerar bajos a moderados si los comparamos con los máximos rendimientos experimentales en la región $\left(27 \mathrm{t} \mathrm{ha}^{-1}\right.$ a $\left.28 \mathrm{t} \mathrm{ha}^{-1}\right)$. Sin embargo, se acercan a los promedios obtenidos en otras investigaciones con materiales del mismo grupo (Rojas y Seminario, 2014; Seminario et al., 2016; Seminario et al., 2017). Es importante anotar la alta variabilidad que muestra el rendimiento de tubérculos:

Así, por ejemplo, en la cosecha II (temporada de estiaje, 2011) el cultivar 'Chachapoyana' y 'Montañera' rindieron $14.7 \mathrm{t} \mathrm{ha}^{-1}$ y $14.2 \mathrm{t} \mathrm{ha}^{-1}$ y el menor rendimiento correspondió a 'Limeña huachuma' con $5.3 \mathrm{t} \mathrm{ha}^{-1}$. Por otro lado, en la cosecha VI (2014), el menor rendimiento ( $\left.3 \mathrm{t} \mathrm{ha}^{-1}\right)$ fue el cultivar 'Porpora' y el más alto $\left(15 \mathrm{t} \mathrm{ha}^{-1}\right)$ correspondió al cultivar 'chachapoyana-1'.

Ambas cosechas se produjeron en época de lluvias, lo que demuestra que el rendimiento es un carácter multifactorial, en el cual, los factores del medio inciden de modo diferenciado en los genotipos. Se puede colegir que los componentes número y peso total de tubérculos por planta y sobre todo el número y peso de tubérculos comerciales por planta, jugaron rol decisivo en la definición del rendimiento total. En general, para todos los cultivares, solo $33 \%$ de tubérculos fueron comerciales pero esta proporción varió de $12.8 \%$ a $66.9 \%$ ) y su peso promedio fue de 37.5 g, pero varió de $21.4 \mathrm{~g}$ a $75.3 \mathrm{~g}$. Asimismo, se puede inferir que las diferencias entre los valores de las variables meteorológicas de las temporadas de lluvia y estiaje jugaron un rol compensatorio entre ellas. Por ejemplo, las menores temperaturas promedio y mínimas de la temporada de estiaje, se compensaron, en cierto modo, con las mayores horas de sol de esta temporada y las menores horas de sol en la época de lluvia, se compensaron con las mayores temperaturas. Es evidente que, en cultivos de campo, es casi imposible encontrar condiciones óptimas (ambientales y de manejo) para las plantas, como lo recomiendan Navas y Díaz (2012). 
Los rendimientos bajos también se explican porque las plantas sólo recibieron una dosis baja de humus $\left(5 \mathrm{t} \mathrm{ha}^{-1}\right)$ y una fertilización de fondo, igualmente en dosis baja (15-24-14 de N-P$\mathrm{K})$. Por otro lado, los antecedentes de estudios experimentales indican que la papa Phureja tiene rendimientos moderados a bajos, inclusive cuando se aplican niveles altos de fertilizante químico o de abono orgánico (Pérez et al., 2008; Muñoz y Lucero, 2008; Gómez-García, 2017). Sin embargo, estos rendimientos se compensan con su precocidad y con el alto precio en el mercado.

Los rendimientos de materia seca en los tubérculos $\left(\mathrm{g}_{\text {planta }}{ }^{-1}\right)$ registrados en los 15 cultivares equivalen a rendimientos de 1 a $2.6 \mathrm{t} \mathrm{ha}^{-1}$ (Figura 1). Estos valores son una expresión del genotipo, debido al diferente porcentaje de materia seca en los tubérculos en cada cultivar, el cual fue determinado durante la caracterización previa (Seminario et al., 2019) y excepto en un caso (cultivar 'Shoga'), los mejores cultivares en peso fresco no fueron los mejores en materia seca (Figura 1). Esta información es importante porque permite comparar y seleccionar cultivares por su eficiencia en el uso de los insumos externos y la energía luminosa y su transformación en materia orgánica, como expresión de la fotosíntesis neta.

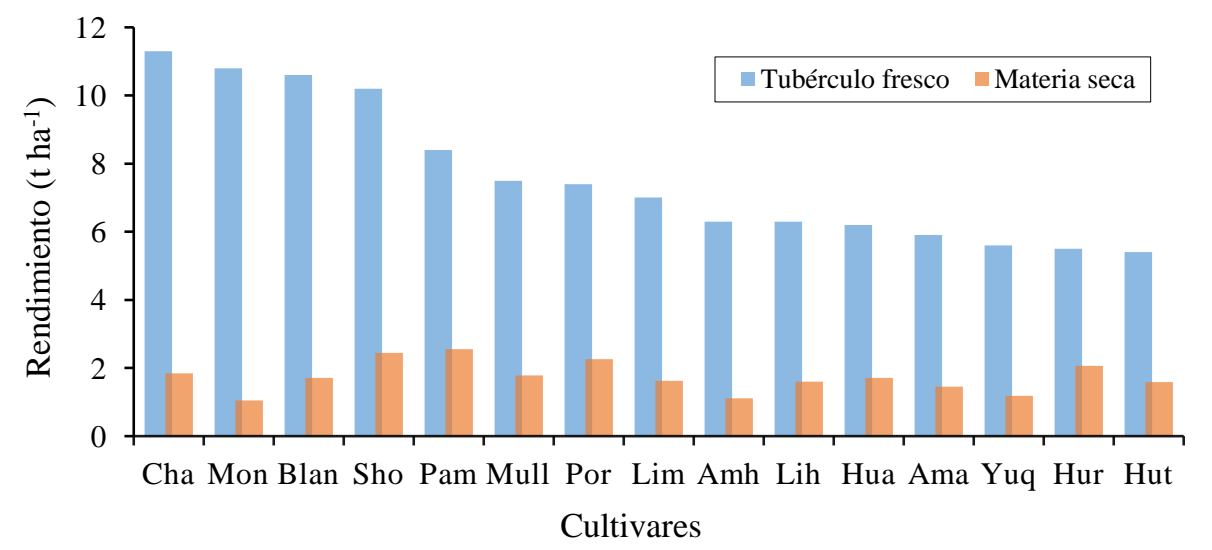

Figura 1. Rendimiento de tubérculos frescos $\left(\mathrm{t} \mathrm{ha}^{-1}\right)$ y de materia seca $\left(\mathrm{t} \mathrm{ha}^{-1}\right)$ de 15 cultivares de papa del grupo Phureja de Cajamarca, en ocho ambientes.

\section{Correlaciones y regresiones entre variables relacionadas con el rendimiento}

Se encontró correlación positiva, altamente significativa, entre PTC y PTT ( $r=0.9387)$ La ecuación de regresión fue: $\mathrm{Y}=55.33+1.2 \mathrm{x}$. Las correlaciones entre NTT, NTC, NT y AP, con PTT fueron bajas y no significativas. La alta correlación positiva entre PTC y el PTT y la falta de correlación entre NT y PTT se explica porque el cultivo produjo abundantes tubérculos pequeños, menores de $2 \mathrm{~cm}$ de diámetro o menores de $5 \mathrm{~g}$ (no comerciales) y los tubérculos comerciales, según la clasificación de Pérez et al. (2008) (comerciales $\geq 2 \mathrm{~cm}$ de diámetro o mayores de $5 \mathrm{~g}$ ) fueron pocos $(30 \% \pm 10 \%$ del total) y fueron los que más influyeron en el peso total de tubérculos por planta (Cuadro 5). Asimismo, la falta de correlación entre NTT, NT y AP con PTT, indica que sus relaciones no son alométricas o estables (Gardner et al., 1985) y por el contrario, estas varían conforme a las condiciones ambientales y de manejo. Rojas y Seminario (2014) analizando 10 cultivares del mismo grupo, también encontraron correlación positiva $(r=0.074)$ entre PTC y PTT. 


\section{Conclusiones}

Se encontraron diferencias estadísticas significativas entre los 15 cultivares tradicionales de papa Phureja en estudio, para las variables PPT, PTC, NTT, NTC, AP, NT y MS. Los cultivares 'Chachapoyana', 'Montañera', 'Blanca' y 'Shoga', fueron los mejores en rendimiento de tubérculos frescos. En cambio, en materia seca destacaron los cultivares 'Piña amarilla', 'Shoga' y 'Porpora' (estos grupos de cultivares deben ser estudiados en pruebas específicas). El componente peso de tubérculos comerciales fue decisivo en la definición del rendimiento total de tubérculos frescos (explicado por su alto grado de correlación, $r=0.93$ ).

Los ambientes (años y meses de siembra diferentes) presentaron diferencias estadísticas significativas paras las variables NTT, NTC, AP y NT. Sin embargo, en general, sus efectos fueron compensatorios, entre sus diversos factores (temperatura promedio, temperatura máxima y mínima, precipitación, humedad relativa y horas de sol) y temporadas, de manera que no se pudo determinar el mejor ambiente.

\section{Literatura citada}

Alarcón, L.; Montoya, K.; Prieto, L. y Cerón, M. 2016. Rehidratación de purés de papa criolla (Solanum tuberosum grupo Phureja). In: Resúmenes del XXVII Congreso de la Asociación Latinoamericana de la Papa. 22-26 agosto, Panamá. 86 p.

Andrade, H. 1997. Requerimientos cualitativos para la industrialización de la papa. Revista Informativa del INIAP. 9:21-23.

Bonierbale, M.; Grüneberg, W.; Amoros, W.; Burgos, G.; Salas, E.; Porras, E. and Felde, T. 2009. Total and individual carotenoid profiles in Solanum phureja cultivated potatoes: II. Development and application of near-infrared reflectance spectroscopy (NIRS) calibration for germoplasm characterization. J. Food Composition and Analysis. 22(6):509-516.

Cerón-Lasso, M.; Alzate-Arbelaez, A. F.; Rojano, B. A. y Ñustez-López, C. E. 2018. Composición fisicoquímica y propiedades antioxidantes de genotipos nativos de papa crioll (Solanum tuberosum grupo Phureja). Información Tecnológica. 29(3):205-216.

Cruz, D.; Sierra, G.; Prieto, L. y Cerón, M. S. 2016. Purés deshidratados de clones candidatos a registro de papa criolla (Solanum tuberosum grupo Phureja). In: Resúmenes del XXVII Congreso de la Asociación Latinoamericana de la Papa. 22-26 agosto, Panamá. 114 p.

Díaz, W. S. y Mosquera, T. 2014. Identificación y cuantificación de carotenoides en una población de Solanum tuberosum grupo Phureja cocida. In: Memorias de XXVI Congreso de la Asociación Latinoamericana de la Papa. 28 septiembre a 2 octubre, 2014, Bogotá, Colombia. $106 \mathrm{p}$.

Gardner, F. P.; Pearce, R. B. and Mitchel, R. L. 1985. Physiology of crop plants. Iowa State University Press, IA, USA. 195-196 pp.

Gómez- García, A. J. 2017. Efecto de la fertilización orgánica e inorgánica, sobre la productividad en el cultivo de papa criolla (Solanum phureja), en la finca Santo Domingo-Municipio de la Calera-Cundinamarca. tesis Administrador Agropecuario. Facultad de Ciencias Agropecuarias, Universidad Nacional de Cundinamarca, Colombia. 113 p.

Haan, S. 2009. Potato diversity at height: multiple dimentions of farmer-driven in situ conservation in the Andes. Ph.D. thesis Wageningen University, The Netherlands. 245 p. 
Hay, R. K. M. and Walker, A. J. 1989. An introduction to the physiology of crop yield. Longman Scientific \& Technical. 190 p.

Ligarreto, G. A. y Suárez, M. N. 2003. Evaluación del potencial de los recursos genéticos de papa criolla (Solanum phureja) por calidad industrial. Agron. Colomb. 21(1-2):83-94.

Martínez, N. M. y Ligarreto, G. A. 2005. Evaluación de cinco genotipos promisorios de papa Solanum tubersoum sp. andigena según desempeño agronómico y calidad industrial Agron. Colomb. 23(1):17-27.

Martínez, P.; Málaga, A.; Betalleluz, I.; Ibarz, A. y Velezmoro, C. 2015. Caracterización funcional de almidones nativos obtenidos de papas (Solanum phureja) nativas peruanas. Sci. Agrop. 6(4):291-301.

Molina, Y.; Caez, G. R.; Rodríguez, M. L.; Cerón, M. S. y Garnica, A. M. 2015. Contenido de antioxidantes en papas criollas nativas (Solanum tuberosum L. grupo Phureja) en proceso de precocción y congelación. Rev. Alimentos Hoy. 23(36):31-41.

Navas, G. E. y Díaz, C. 2012. Criterios para la evaluación y producción de papa criolla para la industria. CORPOICA, Antioquia, Colombia. 48 p.

Parra-Galindo, M. A.; Piñeros-Niño, C. y Mosquera, T. 2016. Composición y cuantificación de antocianinas en tubérculos cocidos de Solanum tubersoum grupo Phureja. In: Resúmenes del XXVII Congreso de la Asociación Latinoamericana de la Papa. 22-26 agosto, Panamá. $76 \mathrm{p}$.

Peña, C.; Restrepo-Sánchez, L. P.; Kushalappa, A.; Rodríguez, L. E.; Mosquera, T. and NarvaezCuenca, C. E. 2015. Nutritional contents of advanced breeding clones of Solanum tuberosum group Phureja. LWT Food Sci. Technol. 62:76-82. Doi:10.1016/j.lwt.2015.01.038.

Pérez, L. C.; Rodríguez, L. E. y Gómez, M. I. 2008. Efecto del fraccionamiento de la fertilización con $\mathrm{N}, \mathrm{P}, \mathrm{K}$ y $\mathrm{Mg}$ y la aplicación de los micronutrientes $\mathrm{B}, \mathrm{Mn}$ y $\mathrm{Zn}$ en el rendimiento y calidad de papa criolla (Solanum phureja) variedad Criolla Colombia. Agron. Colomb. 26(3):477-486.

Piñeros, C. I. 2009. Recopilación de la investigación del sistema de papa criolla. FEDEPAPA, Secretaría de Agricultura y Desarrollo Económico, Gobernación de Cundinamarca, Colombia. $152 \mathrm{p}$.

Rivera, J. E.; Herrera, A. O. and Rodríguez, L. E. 2011. Assessment of the processing profile of six "creole potato" genotypes (Solanum tuberosum Phureja Group). Agron. Colomb. 29(1):73-81.

Rozo, Y. C. and Nustez, C. E. 2011. Effects of phosphorus and potassium levels on the yield of the variety Criolla Colombia in the department of Cundinamarca. Agron. Colomb. 29(2):205-2012.

Rozo, D. y Ramírez, L. N. 2011. La agroindustria de la papa criolla en Colombia. Situación actual y retos para su desarrollo. Gestión Soc. 4(2):17-30.

Saldaña, T. M.; Patiño, J. A. and Cotes-Torres, J. M. 2015. Biomass distribution and allocation in diploid potato varieties (Solanum phureja Juz. et Buk.). Agron. Colomb. 33(3):322-329. Doi: 10.15446/agron.colomb.v33n3.50237.

SAS Institute. 2013. SAS 9.4. Procedure's guide: Statistical procedures. Second edition. SAS Institute Inc. Cary, NC, USA. 550 p.

Seminario, J.; Seminario, A. y Domínguez, A. 2016. Potencial productivo de 12 cultivares de papa chaucha (Solanum tuberosum L. grupo Phureja) de la región Cajamarca. Fiat Lux. 12(2):115-127. 
Seminario, J.; Seminario, A.; Domínguez, A y Escalante, B. 2017. Rendimiento de cosecha de diecisiete cultivares de papa (Solanum tuberosum L.) del grupo Phureja. Sci. Agrop. 8(3):181-191. doi:10.17268/sci.agropecu.2017. 03.01.

Seminario, J.; Villanueva-Guevara, R. y Valdez-Yopla, M. H. 2018. Rendimiento de cultivares de papa (Solanum tuberosum L.) amarillos precoces del grupo Phureja. Agron. Mesoam. 29(3):639-653. Doi: 10.15517/ma.v29i3.32623.

Seminario, J.; Tapia, H. J. y Seminario, A. 2019. Los Solanum del grupo Phureja de Cajamarca: avances. Gráfica Bracamoente. Lima, Perú. 19-61 pp.

Seminario, J. y Zarpán, L. 2011. Conservación in situ on farm-ex situ de Solanum tuberosum L. grupo Phureja en la cuenca del Llaucano y áreas adyacentes. Arnaldoa. 18(2):103-114.

Vásquez, V. 1988. Mejoramiento genético de la papa. Amaru editores, Lima-Perú. 55-56 pp.

Vásquez, V. 2014. Diseños experimentales con SAS. Edita Concytec-Fondecyt. Lima, Perú. 106$107 \mathrm{pp}$.

Zimmerer, K. S. 1991. Labor shortages and crop diversity in the southern Peruvian Serra. Geographical Review. 81(4):414-43. 